Sharif University of Technology
Scientia Iranica
SCIENTIA $\quad \begin{gathered}\text { Transactions D: Computer Science \&s Engineering and Electrical Engineering } \\ \text { http://scientiairanica.sharif.edu }\end{gathered}$

\title{
Massive parallel digital microfluidic biochip architecture for automating large-scale biochemistry assays
}

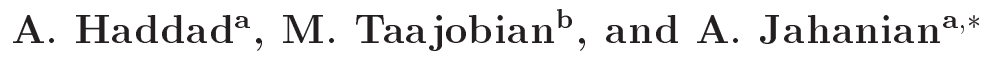 \\ a. Department of Computer Science and Engineering, Shahid Beheshti University, G.C., Tehran, P.O. Box 1982963113, Iran. \\ b. Department of Computer Engineering, Mahdishahr Branch, Islamic Azad University, Mahdishahr, Iran. \\ Received 24 April 2017; received in revised form 3 February 2018; accepted 4 August 2018
}

\section{KEYWORDS}

Microfluidic biochip;

Physical design;

Architecture.

\begin{abstract}
Micro/nanofluidic biochips are used to automate the clinical diagnosis, DNA sequencing, drug discovery, and real-time bio-molecular recognition. One of the attractive usages of biochips is Lab-On-Chip (LOC). Lab-on-Chip technology is a promising replacement for biomedical and chemical apparatus. Two main types of microfluidic-based biochips are used: continuous-flow-based and digital microfluidic biochips (DMFB). In DMFBs, liquids, in the form of droplets, are controlled independently and concurrently over a two-dimensional array of cells (or electrodes). Digital microfluidic biochips are of high ability to configure and for fault tolerance. In this paper, a new architecture for DMFB with an aim of making a balance between the parameters of flexibility, efficiency, cost, and completion time of biological experiments is presented. In the new architecture, a FPGA-based structure is used, which increases flexibility and parallelizing assay operations. Experiments show that the execution times of scheduling, routing, and simulation have improved by about $2.54 \%, 18.76 \%$, and $12.52 \%$, respectively, with $21 \%$ overhead cost in the number of controlling pins.

(C) 2018 Sharif University of Technology. All rights reserved.
\end{abstract}

\section{Introduction}

A biochip is an electronic chip that can be used to control and automate biochemistry reactions. Instead of mixing fluids together based on milliliters and liters in test tubes and beakers, biochips can perform many of the same reactions by manipulating nano-liter-sized quantities of fluid on a small lab-on-chip device. Microfluidic lab-on-chips have been designed to execute a multitude of different biochemical applications, including in-vitro diagnostics and immunoassays, used in clinical pathology and integrate the experience of VLSI and

\footnotetext{
*. Corresponding author. Tel.: +98-21-29904188 E-mail addresses: a_haddad@mail.sbu.ac.ir (A. Haddad); taajobian@msh-iau.ac.ir (M. Taajobian); jahanian@sbu.ac.ir (A. Jahanian).
}

biochemists together in order to simplify and automate the chemical processes and experiments [1-3]. They can bring about a revolution in drug generation and testing, genetic engineering, and clinical diagnostics. Using biochips will reduce time, costs, and laboratory equipment space, significantly occupied $[4,5]$.

There are two different classes of microfluidic biochips: continuous-flow biochips and digital microfluidic biochips. Continuous-flow biochips contain microvalves and micro-pumps to control the flow of a liquid. The other class of biochips is DMFB that includes novel open structures where the liquid is divided into discrete (hence, the name "digital") controllable droplets [6].

Recent advances in digital microfluidic biochips have enabled lab-on-a-chip devices for DNA sequencing, immunoassays, clinical chemistry, and protein crystallization. Basic operations, such as droplet dispensing, mixing, dilution, localized heating, and 
incubation, can be carried out using a two-dimensional array of electrodes and nano-liter volumes of liquid [7].

In a DMFB, activation sequence of electrodes is controlled by a micro-controller, normally based on the target chemical experiment. According to microcontroller pins allocated to electrodes, two categories of addressing methods are proposed: direct addressing and pin-constrained methods. Direct-addressing biochips (DA-DMFB) provide independent control pin over each electrode that increases the flexibility. These devices are very costly (or even infeasible) because the large number of control inputs and high wiring complexity can increase the number of Printed Circuit Board (PCB) layers [8]. Pin-constrained (PC-DMFB) technique was proposed to solve the cost problem in the DA-DMFBs. In the PC-DMFBs, electrodes are grouped, and each cluster has a common external pin. Most important methods of these categories are described in the following sections.

So far, various architectures and algorithms have been presented for the implementation of digital microfludic biochips. However, these architectures have different challenges and face serious problems with flexibility, configuration capabilities, large number of control pins, and long time running bioassays. For example, the application specific architecture is designed for a specific application, and this kind of biochip cannot be generalized to other bioassays [9]. The Generalpurpose [8] and Reconfigurable architectures [10] are also inefficient and have a large number of control pins and, consequently, higher cost. The pin-constrained architecture reduces the pin numbers, yet does not have sufficient flexibility [11]. The field-programmable pinconstrained DMFB architecture [6] is presented as a general-purpose architecture to decrease the number of controlling pins and the corresponding costs, yet lacks the flexibility and requires more time to run large bioassays.

FPGA is a successful platform in microelectronics in terms of flexibility, parallelism, and cost/performance tradeoff. In this paper, a new FPGAinspired architecture is proposed for digital microfluidic biochips to increase the DMFBs in terms of configurability, degree of parallelism, and the chip usability.

The paper is structured as follows. In Section 2, various types of biochips and their architectures are described. In Section 3, proposed architecture and physical design algorithms for digital microfluidic biochip are presented. In Section 4, experimental results are presented, and Section 5 concludes the paper.

\section{An overview of biochips}

A biochip is a collection of miniaturized test sites (microarrays or microfluidic grid) arranged on a solid substrate that provides a platform for performing many chemical tests in order to achieve higher throughput and flexibility. Similar to a computer chip that can perform millions of mathematical operations in one second, a biochip can perform thousands of biological reactions, such as decoding genes in few seconds. Biochips are divided into two categories: microfluidicbased biochips and Microarray-based biochips.

\subsection{Microarray biochips}

The DNA microarray is a piece of glass, plastic or silicon substrate whose DNA pieces are affixed in a microscopic array. These DNA segments act as DNA probes in detecting genetic sequences of a biological sample simultaneously. Similarly, in protein arrays, large quantities of capture agents (like monoclonal antibodies) act as detectors and help determine the presence and/or amount of proteins in biological samples, e.g., blood. GeneChip DNA array from Affymetrix, DNA microarray from Infineon A, and nanochip microarray from nanogen are few DNA microarray technique-based biochips available on the market [2]. A major disadvantage of DNA and protein arrays is that once these chips are synthesized, they are neither configurable nor scalable. Moreover, there is no facility to carry out sample preparation in this kind of biochips.

\subsection{Microfluidic biochips}

In recent years, microfluidic-based biochips have become popular for biochemical analysis. These miniaturized microfluidic-based biochips can perform enzymatic analysis (e.g., glucose, lactate, and private assays of human physiological fluids like saliva, urine, etc.), massive parallel DNA analysis, automated drug discovery, and toxicity monitoring. These biochips can be termed as lab-on-a-chip as it replaces highly repetitive laboratory tasks by replacing cumbrous laboratory equipment with composite micro-system [2].

Mainly, two types of microfluidic-based biochips, i.e., continuous-flow-based and droplet-based microfluidic biochips, are described below.

\subsubsection{Continuous-flow microfluidic biochips}

As mentioned before, the technology of these chips is based on the manipulation of continuous liquid flow through micro-fabricated channels. External pressure sources, integrated mechanical micro-pumps or electrokinetic mechanisms are used for the actuation and manipulation of liquid flow [2].

Continuous flow biochips are useful in carrying out simple biochemical applications, which require less complicated fluid manipulation. Because of the structure of these biochips, it becomes very difficult to integrate and scale these kinds of microfluidic chips. The reconfigurability of these chips is very poor because of the permanent etching of the microstructures. More- 
over, fault tolerance capability is very poor because of the poor reconfigurability of this technology.

\subsubsection{Digital microfluidic biochips}

In digital microfluidic biochips, the liquids in the form of discrete droplets are controlled independently and concurrently over a 2D array of electrodes. The prime advantages of these biochips include reconfigurability, ease of integration, high-level automation, and ability to scale. The assays can be broken down to a few basic operations that can be easily defined and automated. A group of cells can be reconfigured to perform different activities in different phases of the experiment. Due to these properties, biochips can be considered programmable microfluidic processors $[10,11]$.

In DMFBs, droplets can be manipulated using chemical, thermal, acoustical, and electrical principles [12], and there are two kinds of electrical methods for moving the droplets: Di-electrophoresis (DEP) [13,14] and Electro-Wetting On-Dielectric (EWOD) [2]. Figure 1 represents an EWOD-based microfluidic biochip and its basic unit cell. The detailed fabrication process of a basic unit cell in EWOD-based DMFB is described in $[2,7,15]$.

The main fluidic processing carried out on digital microfluidic biochips is as follows $[16,17]$ :

- Creation: To take a certain amount of liquid from a reservoir to form droplets of a given size, Figure 2(a);

- Transport: To move the droplet along a linear path to or from other functional components, such as detectors, catalytic converters and supplies, and waste outlets, Figure 2(b);

- Merging/splitting: To merge droplets and split a droplet into smaller parts for parallel processing, Figure 2(c);

- Mixing: Mixing the contents of a merged or created droplet, Figure 2(d);

- Storage: To store droplets, Figure 2(e).



(a) Creation

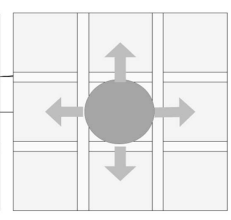

(b) Transporting

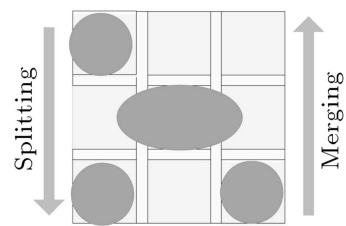

(c) Merging and splitting

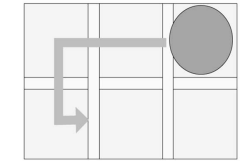

(d) Mixing

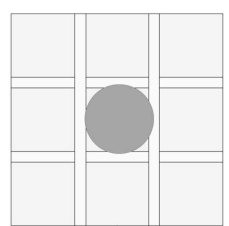

(e) Storage
Figure 2. Basic operations of digital microfluidic biochip [16].

The advantages of DMFBs over huge and heavy systems include design flexibility, higher sensitivity, smaller size, lower cost, less sample size and reagents volume, and lower power consumption.

\section{Various architectures of DMFBs}

Various architectures have been proposed for DMFBs. Since the structure and features of these architectures are effective in the concept of the paper, these architectures are described in the following subsections.

\subsection{Application specific architectures}

In this kind of digital biochips, type and number of modules, their location, and traveling path of the droplets are planned and fixed at the design time for a specific application. It is implied that spatial assignment (location of each operation) and temporal assignment (the activation time of each operation) should be done at the design time. The important aspect in this architecture is that a special chip should be designed for each assay, which is not affordable and reasonable as the price [9]. A sample of these biochips is shown in Figure 3. This biochip is designed

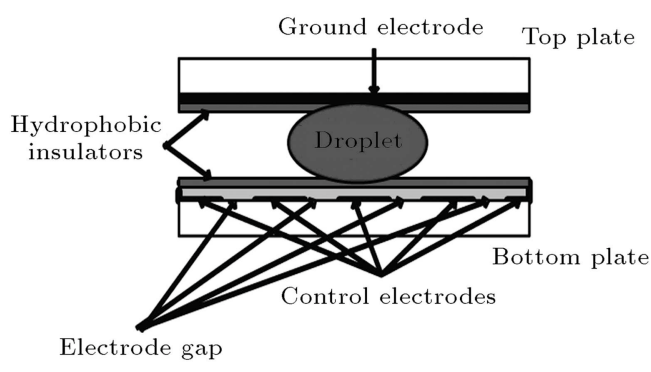

(a)

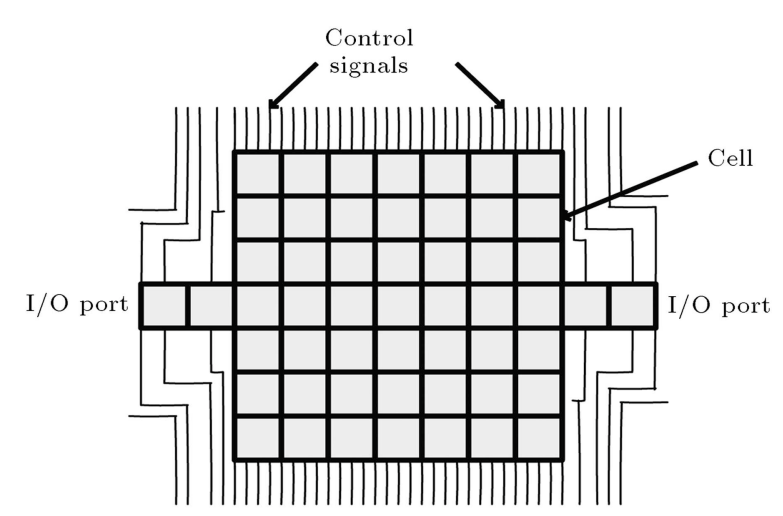

(b)

Figure 1. (a) Basic unit cell in EWOD-based DMFB. (b) A 2-D array for digital microfluidic [15]. 


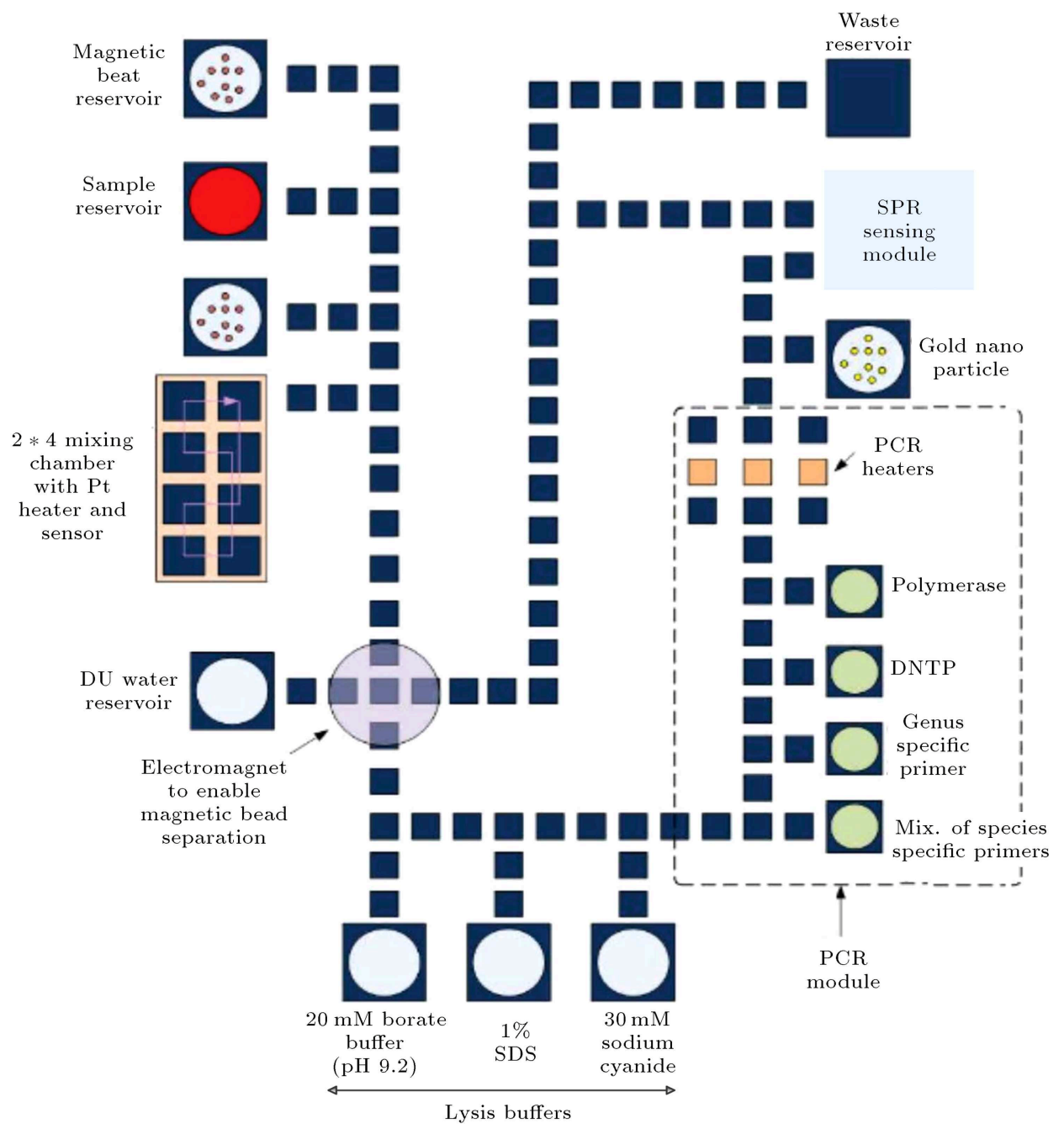

Figure 3. An example of application specific digital biochip for recognition of malaria [9].

and fabricated for a specific application (recognition of Malaria) and cannot be reprogrammed for other applications [9].

\subsection{General-purpose architectures}

In this type of architectures, spatial assignment (location of mix, detect, store, and other modules) should be determined at the design time; however, temporal assignment (scheduling management) should be decided at the usage time. Figure 4 represents a sample of a general-purpose biochip.

As can be seen in Figure 4, routing between modules (dark columns and rows) has been considered at the design time and cannot be changed at the time of executing [8]. The light areas show working parts where only splitting and mixing operations can be done. In this architecture, only splitting and mixing operations can be done in parallel; then, results should be sent to external sources [8].

\subsection{Reconfigurable architectures}

In reconfigurable architectures, place and time attri- butions are determined at the usage time. Position of detect modules and I/O ports should be classified and fixed at the design time; however, the attributions of other modules should be resolved after the design time. Figure 5 shows reconfigurable architecture, where all areas of the chip are covered with electrode, and each one should be controlled by a pin. In this architecture, the electrodes, I/O ports, and detection modules are shown. The main operations, such as mix and store, can be created in every location on two arrays of electrodes [10].

\subsection{Pin-Constrained Digital Microfluidic Biochip (PC-DMFB)}

Increasing the number of electrodes and pins of the previous architectures leads to the increasing number of control pins, PCB (Printed Circuit Board) layers, and cost of these biochips. Therefore, a pin-constrained solution was proposed in $[7,11]$. The basic idea of this solution is to partition electrodes into several groups and connect each group of electrodes to a single control pin. Figure 6(a) and (b) show the direct and pin- 




Figure 4. A simple view of a general-purpose architecture [8].

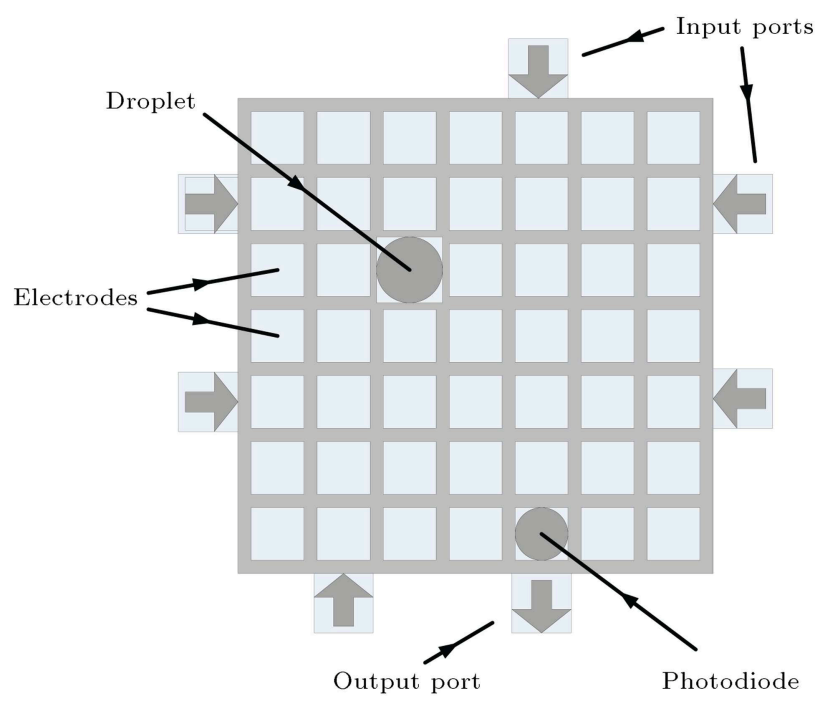

Figure 5. The proposed reconfigurable architecture for digital microfluidic biochips [10].

constrained addressing. In these figures, the same numbers indicate that the corresponding electrodes are connected and controlled by the same pin. Although the number of control pins can be reduced, the pinconstrained solution has low flexibility, because it is usually specific to a microfluidic application.

\subsection{Field-Programmable Pin-Constrained $D M F B$ (FPPC DMFB)}

In [6], a general-purpose architecture is presented to decrease the number of controlling pins and the corresponding costs. In this method, place attribution is done at the design time, while the time attribution is determined at the usage time. Considerable areas of the chip surface are unusable, which are used for module separation. Moreover, I/O sources can be located anywhere in around of the biochip. The problem addressed for this architecture is that larger chips are required for large assays. Figure 7 shows an example of FPPC architecture.

\subsection{Analysis and comparison of architectures}

In the previous section, various types of digital biochip architectures were described in detail. The main advantages of special-purpose biochips include simplicity of design and implementation as well as low production and reagent costs. However, this biochip is application-specific without reconfigurability and programmability capabilities. The general-purpose biochips resolved specific-purpose problems; however, they are not fully-programmable because the locations of modules are pre-designed while routing is done for a specific problem. The next generation of biochips is presented with a fully reconfigurable architecture to improve the flexibility of biochips. This architecture is a two-dimensional array of controllable electrodes, whose electrode is controlled by an external pin; in addition, decreasing the number of pins is very critical in feasibility of biochip construction. For large assays, such devices make huge wiring complexity that requires costly multi-layer PCBs. In contrast, pin-constrained DMFBs reduce the wiring complexity, yet reduce the flexibility of droplet coordination. FPPC DMFBs have been proposed to solve the problems of PC. FPPC 


\begin{tabular}{|c|c|c|c|c|c|c|c|}
\hline 1 & 2 & 3 & 4 & 5 & 6 & 7 & 8 \\
\hline 9 & 10 & 11 & 12 & 13 & 14 & 15 & 16 \\
\hline 17 & 18 & 19 & 20 & 21 & 22 & 23 & 24 \\
\hline 25 & 26 & 27 & 28 & 29 & 30 & 31 & 32 \\
\hline 33 & 34 & 35 & 36 & 37 & 38 & 39 & 40 \\
\hline 41 & 42 & 43 & 44 & 45 & 46 & 47 & 48 \\
\hline 49 & 50 & 51 & 52 & 53 & 54 & 55 & 56 \\
\hline 57 & 58 & 59 & 60 & 61 & 62 & 63 & 64 \\
\hline
\end{tabular}

(a) Direct addressing

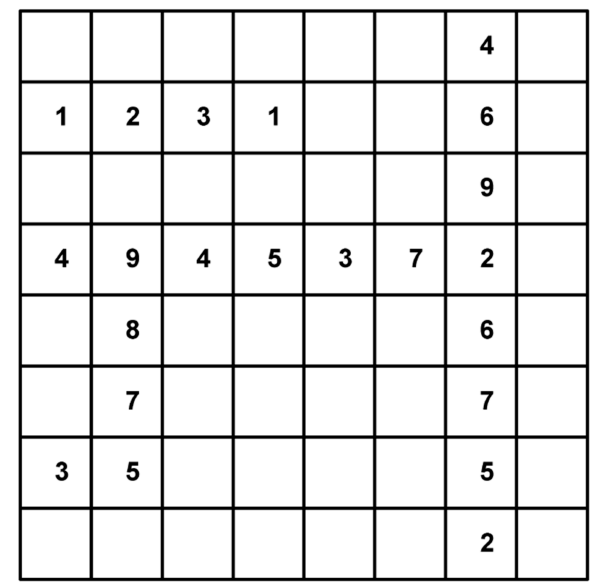

(b) Pin-constrained

Figure 6. Two electrode-addressing solutions: (a) Direct-addressing [8] and (b) pin-constrained [7].

\begin{tabular}{|c|c|}
\hline $14-17$ & Mixer hold \\
\hline $18-21$ & Mixer I/O \\
\hline $22-27$ & Split-store-det I \\
\hline $28-33$ & Split-store-det \\
\hline $7-13$ & Mixing \\
\hline $1-6$ & Routing \\
\hline & Unused space \\
\hline
\end{tabular}

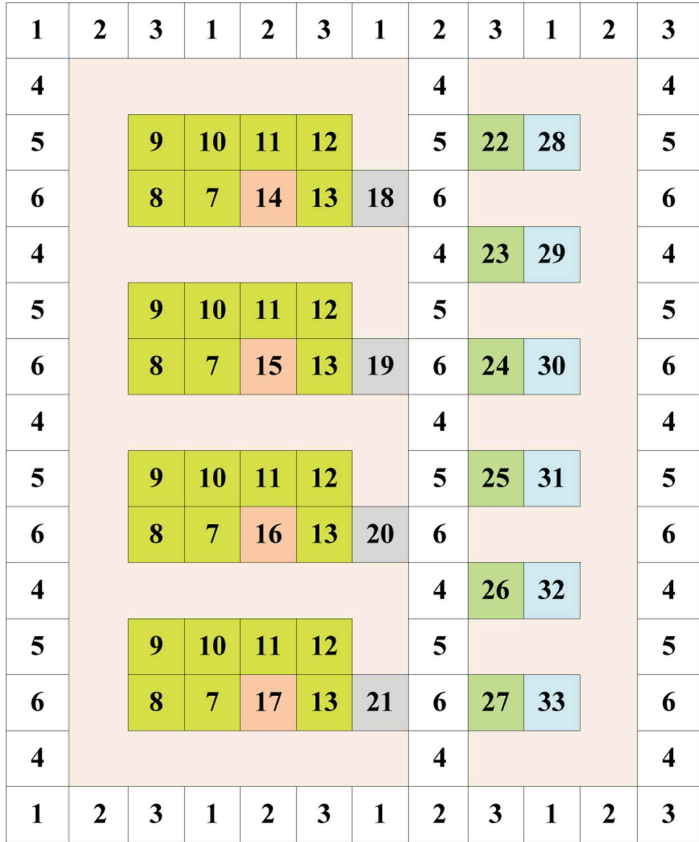

Figure 7. Field-programmable pin-constrained DMFB [6].

DMFBs use a routing column among modules (SSD, MIX,..) to reduce flexibility. In addition, parallelism is impossible.

\section{The proposed architecture and design flow}

As mentioned before, a new architecture is proposed to maintain a reasonable trade-off between efficiency and flexibility. The main objectives of the proposed approach are as follows:

- Reducing the execution time of large-scaled assay operations;

- Parallelizing the operations as much as possible;

- Reducing the number of pins;
- Increasing the flexibility and programmability of microfluidic platform.

In the following subsections, the proposed architecture is described, and then the corresponding design flow is expressed.

\subsection{The proposed architecture}

In the previous sections, various architectures and their problems were mentioned. In this section, a new architecture for microfluidic biochip is presented which is inspirited from the conventional FPGAs. The proposed architecture, which is actually the improved architecture in [18], is induced from FPGA architecture, because FPGAs make a good trade-off between efficiency and flexibility, and using the semantics of 


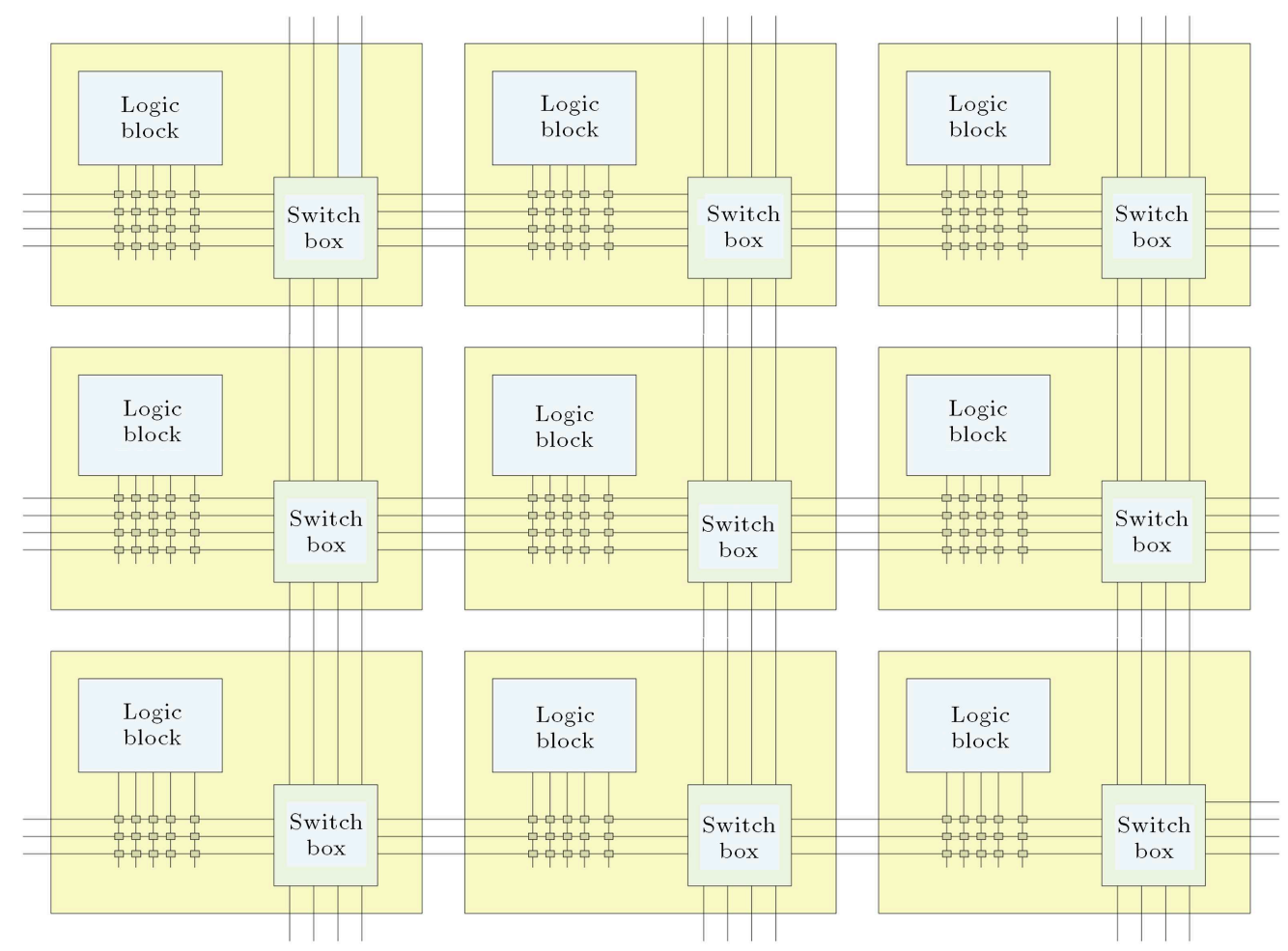

Figure 8. Global architecture of an FPGA.

FPGA architecture in microfluidic biochips will be useful. The proposed architecture can be configured for each assay or biochemical evaluations without new fabrication of the chip. An FPGA contains twodimensional arrays of logic blocks and interconnections between logic blocks. Both the logic blocks and interconnects are programmable. Logic blocks can be programmed to implement the desired functions, and an interconnect is programmed using the switch boxes to realize a specific connection scheme between the logic blocks. General architecture of an FPGA is shown in Figure 8.

The main objective of the proposed architecture is to increase the flexibility and parallelism of the biochips. Figure 9 shows the proposed architecture.

The presented architecture in Figure 9 is a twodimensional array of Configurable Bio-Cell (CBC). Each $\mathrm{CBC}$ is analogues to FPGA logic block that contains the primary operations of the biochemical treatments. In this architecture, input and output ports of chemical material are the same as the FPGA I/O pins too. The electrodes are used to control the droplet routing that travels among the CBCs. This architecture is called Programmable Bio-Cell Matrix (PBCM). Figure 10 shows the internal structure of a $\mathrm{CBC}$ that contains the following elements:

- A mixing module that is specialized with electrodes $7-13$;
- Input-output port of mixing module or I/O mixer that is specialized with electrodes 16-17;

- Mixer hold module that is specialized with electrodes 14-15;

- Three Split-Store-Detection (SSD) modules that are electrodes 21-23;

- Input-output port of SSD modules or I/O SSD that are electrodes 18-20;

- Routing electrodes (1-6).

In Figure 10, electrodes 1-3 of each cell are used to transfer droplets in horizontal buses, and electrodes 4-6 are used to transfer droplets in vertical buses.

These electrodes should be indexed in all the CBCs in the same order because all electrodes of the CBCs with the same index are connected to one controlling pin. In this structure, the sameindex electrodes are activated simultaneously with one controlling pin. Electrodes 7-14 should create a mixing unit, and droplets which should be mixed must enter and mix in these units from various routes.

Electrodes 7-13 are common in all cells and are activated at the same time; however, electrode 14 is used to store a mixed droplet in each cell and is controlled with different pins in various cells. Electrode 16 is input-output ports for mixing module, which is used to enter or exit droplet. It should be controlled with different pins in various cells. This ability should exist so that one droplet in a cell can exit mixing 


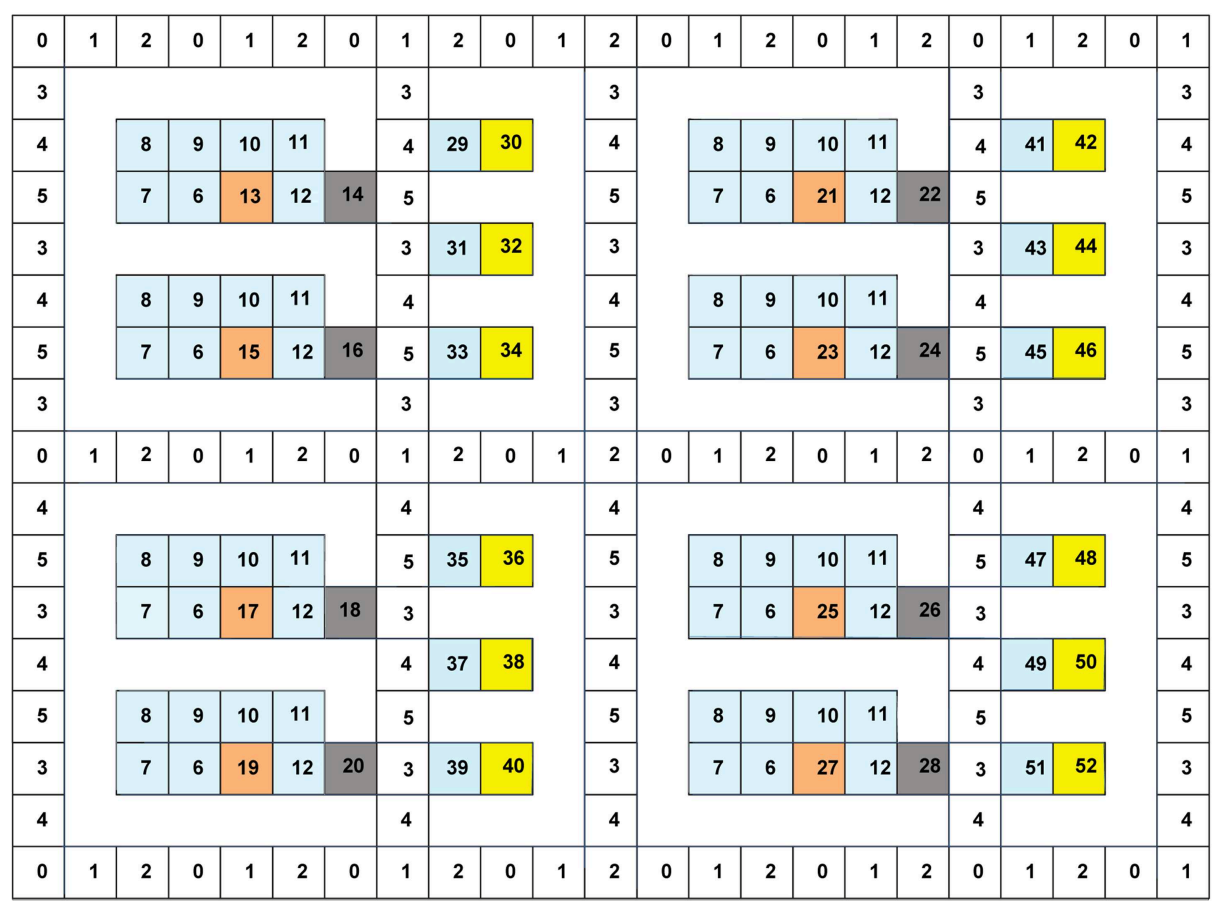

Figure 9. A simple view of a PBCM with 4 CBCs.

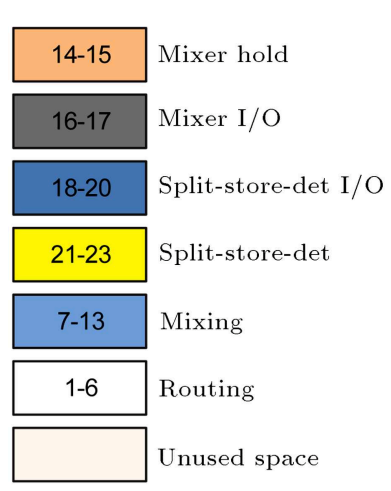

\begin{tabular}{|c|c|c|c|c|c|c|c|c|c|c|c|}
\hline 1 & 2 & 3 & 1 & 2 & 3 & 1 & 2 & 3 & 1 & 2 & 3 \\
\hline 4 & & & & & & & 4 & & & & 4 \\
\hline 5 & & 9 & 10 & 11 & 12 & & 5 & 18 & 21 & & 5 \\
\hline 6 & & 8 & 7 & 14 & 13 & 16 & 6 & & & & 6 \\
\hline 4 & & & & & & & 4 & 19 & 22 & & 4 \\
\hline 5 & & 9 & 10 & 11 & 12 & & 5 & & & & 5 \\
\hline 6 & & 8 & 7 & 15 & 13 & 17 & 6 & 20 & 23 & & 6 \\
\hline 4 & & & & & & & 4 & & & & 4 \\
\hline 1 & 2 & 3 & 1 & 2 & 3 & 1 & 2 & 3 & 1 & 2 & 3 \\
\hline
\end{tabular}

Figure 10. Internal structure of a Configurable Bio-Cell (CBC).

module, while other droplets remain in other cells for some time steps.

Electrodes 21-23 are used for storing, splitting, and detecting droplets and are controlled with different pins in various cells. If, during executing assays, droplets need to be detected by external sources; these detectors should be located above SSD electrodes and detect droplets in some time steps. Storing operation requires a droplet to enter a SSD module (for example, electrode 21) and remain in place. According to Figure 10, for splitting operation, the initial position of droplet, which will be split, is on a vertical transport bus next to an SSD modules I/O cell (for example, electrode 5). The cell on the transport bus is activated throughout the split. The I/O cell (electrode 18) is then activated, which stretches droplet to cover both cells.
Next, the SSD modules' hold cell is activated, and the I/O cell is deactivated; this splits the droplet into two separate droplets on the hold cell and in the transport bus. Connections between SSD modules and vertical routes should be made by electrodes $18-20$, which are controlled with different pins in various cells.

Electrodes connecting cells with a circular area and connecting electrodes between internal cells can all be controlled via common pins to transfer droplets between cells without overlapping.

In Figure 10, a PBCM with 4 cells is shown whose CBCs have a similar structure to those in Figure 8 in which all the CBCs are connected together using the routing paths. Input/output sources and dispensers are located around the chip. Regarding the above explanations, the biochip of Figure 10 can perform 


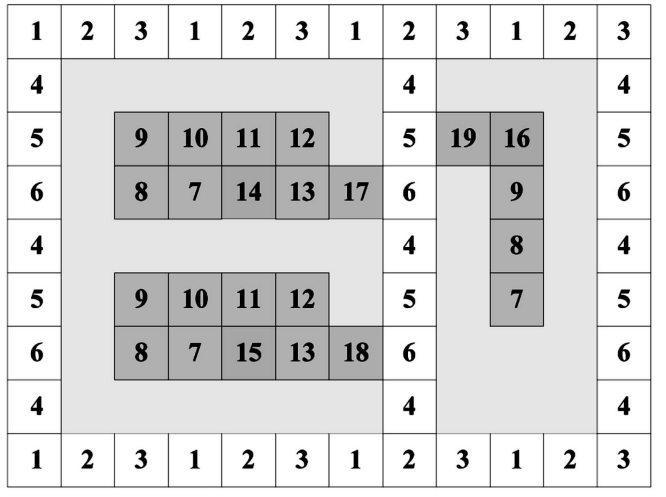

(a)

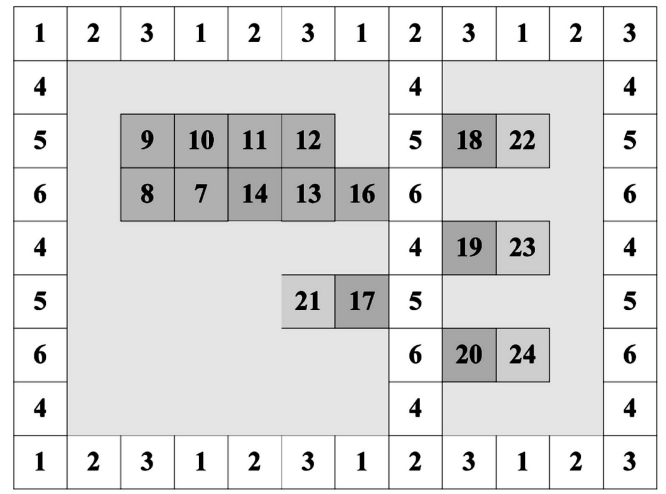

(b)

Figure 11. (a) CBC with MIX modules. (b) CBC with a single MIX and many SSD modules.

parallel 8 mixes, 12 splits, and store operations on minimum 20 droplets. This plan is useful for large assays with lots of operations because the time of assays can be shortened by parallelizing the operations. All the MIX modules have been initialized with identical pin numbers so that all MIX operations on the chip surface can be run simultaneously, leading to the parallelization of the MIX operation. On the other side, for small assays or the ones that do not need all the cells, extra cells can be deactivated to reduce power consumption.

It is worth noting that the internal structure of CBCs can be customized for target assays. For example, CBCs can be considered with more MIX modules for the assays with a large number of MIX operations (Figure 11(a)), and they can be designed with more number of SSD modules when the assays have a large number of SSD operations, as shown in Figure 11(b).

\subsection{Design flow for the proposed architecture}

Input of a microfluidic biochip CAD flow is an assay. This assay is broken down to a few basic operations and converted to a Directed Acyclic Graph (DAG) that can be easily defined and automated, normally. In assay DAG, nodes represent the set of operations such as merge, MIX, split, transport, detect, etc., and edges show the droplet transfer between the operation modules. For example, sequencing graph for the mixing stage of PCR with 7 MIX modules is shown in Figure 12 [19].

Static Synthesis Simulator (SSS) [20] is a widely used academic synthesis framework developed at UC Riverside to develop and evaluate the CAD modules for digital microfluidic biochips [14]. SSS is a full synthesis flow (e.g., scheduling, placement, routing) of assay graphs with simulation and GUI toolboxes. We revised SSS according to our architecture. The main part of SSS that should be updated for a new

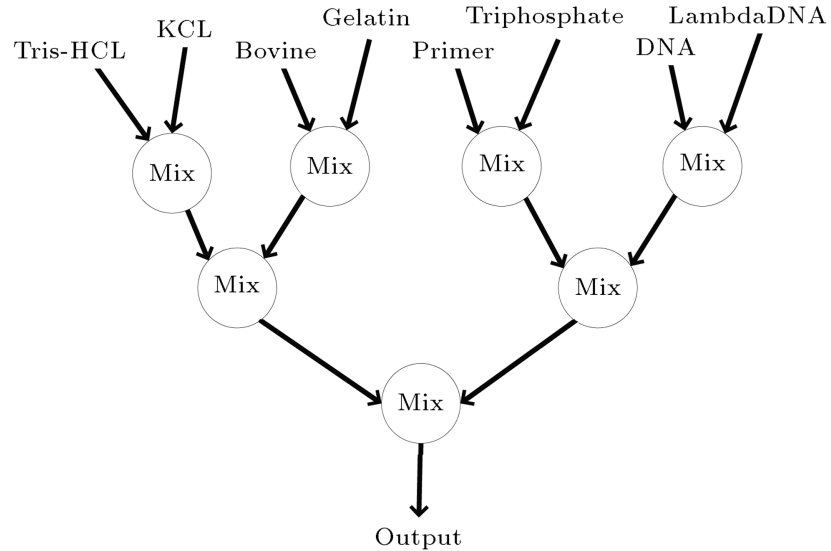

Figure 12. Sequencing graph for the mixing stage of PCR [19].

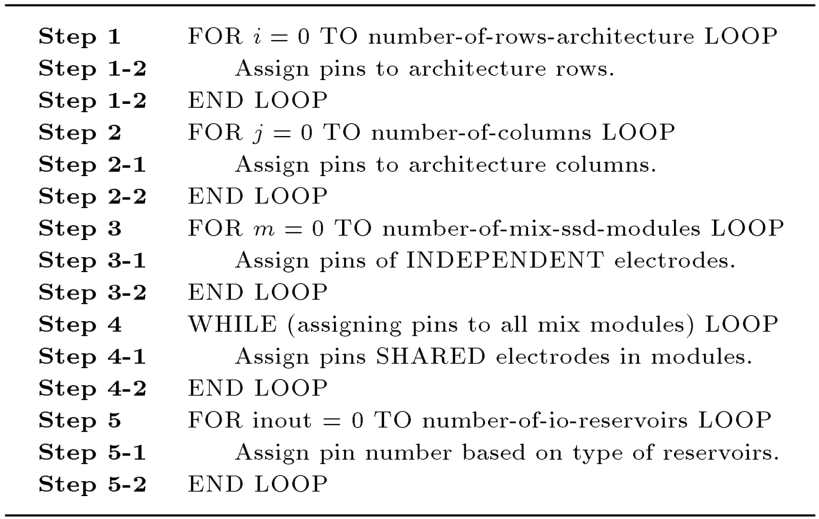

Figure 13. PBCM DMFB pin mapping algorithm.

architecture is pin mapping module. Pseudo code of the pin-mapping algorithm is shown in Figure 13.

In the following paragraphs, detailed description of the pin-mapping algorithm will be described:

Step 1: Pin numbers will be assigned to the electrodes in the rows between the biochip modules;

Step 2: Pin numbers will be assigned to the electrodes in columns between biochip modules; 


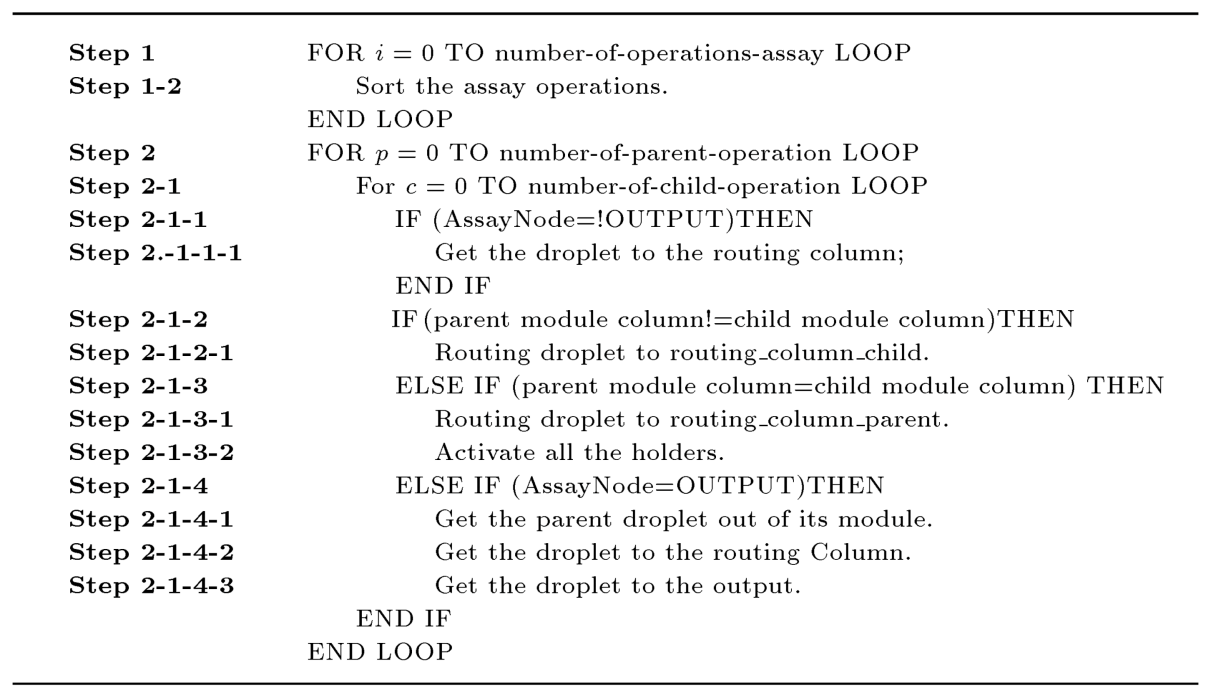

Figure 14. PBCM DMFB routing algorithm.

Step 3: Unique pin numbers will be assigned to the electrodes of the MIX and the detection modules;

Step 4: Pin numbers 7 to 13 will be shared between all MIX modules and assigned to the corresponding electrodes;

Step 5: Pin number of reservoirs' I/O electrodes that are connected to the chip will be assigned.

Another part of SSS that should be revised for the proposed architecture is routing algorithm. Figure 14 represents the revised routing algorithm.

The input of this algorithm is a sequencing graph of the bioassay that is generated in the first stage of synthesis flow, and the output is a sequence of pinactivating process to specify the path of the droplets. The sequencing graph describes the nodes and their relations in the bioassay to the assay rules. In fact, the routing stage determines how the droplets move between the placed modules on the chip surface. In the following paragraphs, a detailed description of the PBCM DMFB routing algorithm will be described:

Step 1: Sort the operations of the assay. Get the nodes that need to be routed; then, sort and get dependencies between nodes.

Step 2: Perform actual routing of droplets based on the number of nodes in the original (parent).

Step 2-1: Do actual routing of droplets primarily based on the number of subnodes (child).

Step 2-1-1: If the node is not OUTPUT module (it is input or basic module), the droplet should exit the module and enter the column. Routing is connected to the module (the electrodes numbered by $0-2$ in 9 ).

Step 2-1-2: If the child's routing column is not the same as the column number of the parent module, droplet should be routed from its module to the column of child's module.

Step 2-1-3: If the child's column is the same as the column number of the parent module, droplet moves in the routing column to achieve destination module. In addition, the hold pin of all compound modules will be active.

Step 2-1-4: At this point, the routing is fed to the output reservoir.

\section{Experimental results}

The proposed microfluidic architecture in SSS toolkit $[17,21]$ is implemented to evaluate the efficiency of this architecture. The proposed architecture is compared with the presented biochip in [8] based on direct-addressing (DA) of electrodes with controlling pins and addressed method in [6] based on the programmable method with considering pin-constrained (FPPC). The proposed method in this paper is called PBCM in experimental results. We have used $2 * 2$ CBC structure to execute assays. Various metrics are considered that can be classified in three groups: fabrication cost, timing, and resource usage. A comparison of the presented architecture and design flow in terms of metrics is described in the following subsections.

\subsection{Fabrication cost}

In this subsection, the proposed architecture and design flow can be evaluated in terms of the number of pins and electrodes that are effective in area and cost of microfluidic biochip. Comparison parameters for this phase include maximum area of microfluidic chip (dimension of microfluidic matrix), number of electrodes used in the array, and the number of control pins. 
Fourteen different assays are selected as benchmarks for better evaluation.

As mentioned before, the number of pins is the most significant limitation in microfluidic biochips, and reducing the number of controlling pins is an important challenge. In Table 1, the proposed architecture is compared in terms of the number of control pins. In

Table 1. Comparison of the suggested method with $[6,8]$ in terms of the number of control pins.

\begin{tabular}{lccc}
\hline \multirow{2}{*}{ Assays } & \multicolumn{3}{c}{ \#Pins } \\
\cline { 2 - 4 } & DA & FPPC & PBCM \\
\hline PCR & 285 & 33 & 33 \\
Multi PCR & 285 & 33 & 33 \\
In-vitro 1 & 285 & 43 & 43 \\
In-vitro 2 & 285 & 43 & 43 \\
In-vitro 3 & 285 & 43 & 43 \\
In-vitro 4 & 285 & 43 & 43 \\
Protein & 285 & 75 & 53 \\
ProteinSplit1Eq & 285 & 75 & 53 \\
ProteinSplit2Eq & 285 & 75 & 53 \\
ProteinSplit3Eq & 285 & 75 & 53 \\
ProteinSplit4Eq & 285 & 75 & 53 \\
ProteinSplit5Eq & 285 & 75 & 53 \\
ProteinSplit6Eq & 285 & 75 & 53 \\
ProteinSplitFT & 375 & 75 & 53 \\
Average & $\mathbf{2 9 1 . 4 2}$ & $\mathbf{5 9 . 8 5}$ & $\mathbf{4 7 . 2 8}$ \\
Improve to FPPC & & $\mathbf{2 1 \%}$ & \\
Improve to DA & & $\mathbf{8 3 . 7 \%}$ & \\
\hline
\end{tabular}

this table, column '\#Pins' represents the number of control pins, and the last 2 rows show the percent of reduction in the number of pins compared with DA and FPPC architectures.

As can be seen in Table 1, the number of required pins in the proposed architecture is improved by $83.7 \%$ and $21 \%$ as compared to DA and FPPC methods, alternatively. Results of this table represent that, in the proposed architecture, better functionality and higher flexibility can be achieved with a few number of physical pins (on average). Increasing the number of PCB layers always accompanied by high costs and fewer number of control pins in the PBCM architecture reduces manufacturing costs of biochips considerably.

Table 2 compares the chip area (dimension) and number of electrodes in the proposed architecture (PBCM) with existing architectures (DA and FPPC). In this table, column array dimension shows the number of row and columns in the biochip, and column \#UE represents the number of used electrodes. The last two columns represent the improvement of the number of used electrodes of BPCM rather than conventional methods (DA and $\mathrm{PBCM}$ ).

As can be seen in Table 2, the number of required electrodes in the proposed architecture is improved by $22.69 \%$ as compared to DA, but increased by $4.3 \%$ compared to FPPC. A higher level of flexibility of $\mathrm{PBCM}$ is earned with $22.69 \%$ improvement in electrodes as compared to DA and $4.3 \%$ compared with FPPC. Moreover, the most significant cost of a microfluidic biochip is the number of pins, and overhead in the number of electrodes is more tolerable for designers.

Table 2. Comparison of the suggested method with [6,8] in terms of the number of electrodes and the dimensions.

\begin{tabular}{|c|c|c|c|c|c|c|}
\hline \multirow{2}{*}{ Assays } & \multicolumn{3}{|c|}{ Array dimension } & \multicolumn{3}{|c|}{ \#UE } \\
\hline & DA & FPPC & PBCM & DA & FPPC & PBCM \\
\hline PCR & $15 * 19$ & $12 * 15$ & $23 * 17$ & 285 & 111 & 235 \\
\hline Multi PCR & $15 * 19$ & $12 * 15$ & $23^{*} 17$ & 285 & 111 & 235 \\
\hline In-vitro 1 & $15 * 19$ & $12 * 21$ & $23 * 17$ & 285 & 153 & 235 \\
\hline In-vitro 2 & $15^{*} 19$ & $12 *_{21}$ & $23 * 17$ & 285 & 153 & 235 \\
\hline In-vitro 3 & $15 * 19$ & $12 * 21$ & $23^{*} 17$ & 285 & 153 & 235 \\
\hline In-vitro 4 & $15 * 19$ & $12 *_{21}$ & $23 * 17$ & 285 & 153 & 235 \\
\hline Protein & $15 * 19$ & $12 * 41$ & $23 * 17$ & 285 & 290 & 235 \\
\hline ProteinSplit1Eq & $15 * 19$ & $12 * 41$ & $23 * 17$ & 285 & 290 & 235 \\
\hline ProteinSplit2Eq & $15 * 19$ & $12 * 41$ & $23 * 17$ & 285 & 290 & 235 \\
\hline ProteinSplit3Eq & $15 * 19$ & $12 * 41$ & $23 * 17$ & 285 & 290 & 235 \\
\hline ProteinSplit4Eq & $15 * 19$ & $12 * 41$ & $23 * 17$ & 285 & 290 & 235 \\
\hline ProteinSplit5Eq & $15 * 25$ & $12 * 41$ & $23 * 17$ & 375 & 290 & 235 \\
\hline ProteinSplit6Eq & $15 * 25$ & $12 * 41$ & $23 * 17$ & 375 & 290 & 235 \\
\hline ProteinSplitFT & $15 * 25$ & $12 * 41$ & $23 * 17$ & 375 & 290 & 235 \\
\hline Average & & & & 304.28 & 225.28 & 235 \\
\hline Improve \#UE to FPPC & & & & & $-4.3 \%$ & \\
\hline Improve \#UE to DA & & & & & $22.69 \%$ & \\
\hline
\end{tabular}


Table 3. Comparison of the suggested method with [6] in terms of the execution time of scheduling, routing, and simulation algorithms.

\begin{tabular}{|c|c|c|c|c|c|c|}
\hline \multirow{2}{*}{ Assays } & \multicolumn{2}{|c|}{ Scheduling time } & \multicolumn{2}{|c|}{ Routing time } & \multicolumn{2}{|c|}{ Simulation time } \\
\hline & FPPC & PBCM & FPPC & PBCM & FPPC & PBCM \\
\hline PCR & 3 & 1 & 8 & 4 & 35 & 24 \\
\hline Multi PCR & 6 & 2 & 12 & 8 & 69 & 76 \\
\hline In-vitro 1 & 7 & 7 & 19 & 21 & 103 & 426 \\
\hline In-vitro 2 & 11 & 11 & 25 & 28 & 163 & 204 \\
\hline In-vitro 3 & 22 & 22 & 32 & 37 & 443 & 574 \\
\hline In-vitro 4 & 32 & 29 & 43 & 46 & 705 & 992 \\
\hline Protein & 37 & 34 & 261 & 211 & 4098 & 3094 \\
\hline ProteinSplit1Eq & 8 & 7 & 104 & 80 & 574 & 413 \\
\hline ProteinSplit2Eq & 16 & 15 & 156 & 123 & 1537 & 1123 \\
\hline ProteinSplit3Eq & 36 & 34 & 263 & 209 & 4105 & 3093 \\
\hline ProteinSplit4Eq & 79 & 77 & 482 & 385 & 11342 & 9006 \\
\hline ProteinSplit5Eq & 179 & 178 & 926 & 746 & 33936 & 28567 \\
\hline ProteinSplit6Eq & 411 & 408 & 1823 & 1480 & 113558 & 101936 \\
\hline ProteinSplitFT & 15 & 15 & 160 & 127 & 1521 & 1110 \\
\hline Average & 61.57 & 60 & 308.14 & 250.35 & 12299 & 10759 \\
\hline Average & \multicolumn{2}{|c|}{$2.54 \%$} & \multicolumn{2}{|c|}{$18.76 \%$} & \multicolumn{2}{|c|}{$12.52 \%$} \\
\hline
\end{tabular}

\subsection{Timing}

In the second stage of experiments, the proposed architecture has been examined and compared from the timing perspective. Comparison parameters for this phase include routing delay, scheduling delay, and simulation time. Table 3 shows the results of scheduling, routing, and simulation times for two PBCM and FPPC architectures.

As shown in Table 3, the timing parameters are better for the proposed architecture than those for FPPC. This improvement resulted from the parallelism capability in PBCM. In the proposed method, merging, split, and detection operations are done separately in distinct modules and blocks; however, all of them operate in parallel with the same pins. In each assay, related droplets with related operations enter a CBC block and are stored in SSD modules on the same CBC blocks after performing the operations. In addition, droplets are kept in CBC blocks and moved to other CBC blocks to merge with other droplets or split to carry out other operations or detection, if necessary. Therefore, the number of droplets able to carry out parallel operations in the entire DMFB will increase.

Since this chip does some mixing and operations are detected separately and in parallel, the execution time of assays, especially huge assays, will decrease. On the other hand, our proposed biochip can run any assay, especially assays with a large number of
MIX operations on one chip, and it is not necessary to increase dimension of chip for larger assays. In addition, our method can reduce power consumption, because, for small assays, the unused electrodes or CBC blocks can be off.

According to Table 3, the execution time of routing and simulation algorithms worsened for in-vitro assays in PBCM architecture due to their low number of operations. However, it is more efficient for protein assays (protein assays consist of many detection and MIX operations). As a result, PBCM architecture has higher efficiency for assays with a large number of operations.

\subsection{Resource usage}

In the last phase of experiments, the proposed architecture is studied from resource consumption perspective. Table 4 shows the experimental results in terms of the used resources (SSD and MIX modules) for the attempted benchmarks in FPPC and PBCM architectures.

As can be seen in Table 4, the number of used SSD and MIX modules is reduced for the proposed architecture in comparison with FPPC architecture by about $25.07 \%$ and $28.01 \%$, respectively. As a result, $\mathrm{PBCM}$ architecture is used to optimize MIX and SSD modules. 
Table 4. Comparison of the suggested method with [6] in terms of the number of MIX and SSD modules.

\begin{tabular}{|c|c|c|c|c|}
\hline \multirow{2}{*}{ Assays } & \multicolumn{2}{|c|}{ \#MIX } & \multicolumn{2}{|c|}{ \#SSD } \\
\hline & FPPC & PBCM & FPPC & PBCM \\
\hline PCR & 4 & 4 & 6 & 6 \\
\hline Multi PCR & 4 & 4 & 6 & 6 \\
\hline In-vitro 1 & 6 & 4 & 9 & 6 \\
\hline In-vitro 2 & 6 & 4 & 9 & 6 \\
\hline In-vitro 3 & 6 & 8 & 9 & 12 \\
\hline In-vitro 4 & 6 & 8 & 9 & 12 \\
\hline Protein & 12 & 8 & 19 & 12 \\
\hline ProteinSplit1Eq & 12 & 8 & 19 & 12 \\
\hline ProteinSplit2Eq & 12 & 8 & 19 & 12 \\
\hline ProteinSplit3Eq & 12 & 8 & 19 & 12 \\
\hline ProteinSplit4Eq & 12 & 8 & 19 & 12 \\
\hline ProteinSplit5Eq & 12 & 8 & 19 & 12 \\
\hline ProteinSplit6Eq & 12 & 8 & 19 & 12 \\
\hline ProteinSplitFT & 12 & 8 & 19 & 12 \\
\hline Average & 9.142 & 6.85 & 14.28 & 10.28 \\
\hline Improve to FPPC & \multicolumn{2}{|c|}{$25.07 \%$} & \multicolumn{2}{|c|}{$28.01 \%$} \\
\hline
\end{tabular}

\section{Conclusion}

In this paper, a new architecture was proposed to perform basic operations of biochips with the lower number of control pins that can implement any assay with parallel operations. In general, biochips with the proposed architecture are programmable for all assays, and it is not necessary to increase dimension of biochip for larger assays. In addition, the flexibility of biochips could increase by changing the internal structure of CBCs.

Another advantage of the proposed architecture is that with parallelism of operations, the execution time of assay was reduced, especially for large assays with a large number of MIX and detection modules. In this architecture, the time consumption was reduced by about $12.52 \%$, while the lower number of electrodes was used in comparison with FPPC method. In the proposed structure, the assays performed using several rows and columns between modules for routing, and this method increased the reliability of biochips. In addition, the number of required pins in the proposed architecture decreased by $83.7 \%$ and $21 \%$ in comparison with DA and FPPC methods, respectively. The proposed method decreased layers of PCB by reducing the number of control pins in comparison with previous architectures; thus, this method reduced the construction cost of biochips.

The biggest limitation of this design increased the size of the chip and increased the number of electrodes, resulting in a slight increase in the fixed cost of the chip. Of course, the increase of the cost was due to the increase of the area of the chip, not the number of PCB layers.

\section{References}

1. Iliescu, C., Taylor, H., Avram, M., Miao, J., and Franssila, S. "A practical guide for the fabrication of microfluidic devices using glass and silicon", Journal of Biomicrofluidics, 6(2), pp. 16505-16516 (2012).

2. Zeng J. "Design automation methods and tools for microfluidics-based biochips", In Electronics and Electrical Engineering, Springer, Netherlands (2006).

3. Srinivasan, V., Pamula, V., and Fair, R. "An integrated digital microfluidic lab-on-a-chip for clinical diagnostics on human physiological fluids", Lab on a Chip Journal, 4(5), pp. 310-315 (2004).

4. Sista, R., Hua, Z., Thwar, P., Sudarsan, A., Srinivasan, V., Eckhardt, A., Pollack, M., and Pamula, V. "Development of a digital microfluidic platform for point of care testing", Lab on a Chip Journal, 8(12), pp. 20912104 (2008).

5. Su, F. and Chakrabarty, K. "High-level synthesis of digital microfluidic biochips", ACM Journal on Emerging Technologies in Computing Systems, 3(4), pp. 16-48 (2008). 
6. Grissom, D. and Brisk, P. "A field-programmable pinconstrained digital microfluidic biochip", ACM/IEEE Design Automation Conference, pp. 1-9 (2013).

7. Xu, T. and Chakrabarty, K. "Broadcast electrodeaddressing for pin-constrained multi-functional digital microfluidic biochips", Proceedings of Design Automation Conference, pp. 173-178 (2008).

8. Griffith, E.J. and Akella, S. "Coordinating multiple droplets in planar array digital microfluidic system", International Journal of Robotics Research, 4(11), pp. 933-949 (2005).

9. Dhar, S., Drezdon, S., and Maftei, E. "Digital microfluidic biochip for malaria detection", Technical Report, Duke University (2008).

10. Maftei, E., Pop, P., and Madsen, J. "Tabu searchbased synthesis of dynamically reconfigurable digital microfluidic biochips", Proceedings of the International Conference on Compilers, Architecture, and Synthesis for Embedded Systems, pp. 195-204 (2009).

11. Xu, T. and Chakrabarty, K. "Droplet-trace-based array partitioning and a pin assignment algorithm for the automated design of digital microfluidic biochips", Proceedings of IEEE/ACM CODES+ISSS, pp. 112-117 (2006).

12. Gupta, M.N. "Multi-Board digital microfluidic biochip synthesis with droplet crossover optimization", MSc Thesis, University of Cincinnati (2014).

13. Su, F., Chakrabarty, K., and Fair, R.B. "Microfluidicsbased biochips technology, issues, implementation platforms, and design-automation challenges", IEEE Transactions on Computer-Aided Design of Integrated Circuits and Systems, 25(2), pp. 211-223 (2006).

14. Jones, T.B. "Dielectrophoretic liquid actuation and nanodroplet formation", Application Physicl Journal, 89(2), pp. 1441-1448 (2001).

15. Pollack, M.G. "Electrowetting-based microactuation of droplets for digital microfluidics", PhD. Thesis, Duke University (2001).

16. Chakrabarty, K. "Digital microfluidic biochips: A vision for functional diversity and more than moore", International Conference on VLSI Design, pp. 452-457 (2010).

17. Grissom, D. and Brisk, P. "Fast online synthesis of generally programmable digital microfluidic biochips", In Proceedings of CODES+ISSS, pp. 413-422 (2012).
18. Taajobian, M. and Jahanian, A. "Higher flexibility of reconfigurable digital micro/nano fluidic biochips using an FPGA-inspired architecture", Scientia Iranica. Transactions F, Nanotechnology, 23(3), pp. 1554-156 (2016).

19. Maftei, E. "Synthesis of digital microfluidic biochips with reconfigurable operation execution", $\mathrm{PhD}$ Thesis, Technical University of Denmark (2011).

20. Grissom, D. "Design of topologies for interpreting assays on digital microfluidic biochips", PhD. Thesis, Riverside: University of California (2014).

21. Grissom, D. and Brisk, P. "A high-performance online assay interpreter for digital microfluidic biochips", In The Proceedings of Great Lakes Symposium on VLSI, pp. 103-106 (2012).

\section{Biographies}

Abbas Haddad received BSc degree in Computer Engineering from University of Khayyam in 2011. In 2014, he completed his MSc degree at Shahid Beheshti University.

Maryam Taajobian received BSc degree in Computer Engineering from Tehran University in 2004 and in 2007. She received his MSc degree from the Amirkabir University of Technology after having researched in electronic design automation and physical design of chips. Currently, she is a PhD student at the Department of Computer Engineering at Science and Research Branch of Islamic Azad University, Tehran, Iran. Her research has centered on design of digital microfluidic biochips.

Ali Jahanian received the BSc degree in Computer Engineering from University of Tehran, Tehran, Iran in 1996 and the MSc and PhD degrees in Computer Engineering at Amirkabir University of Technology, Tehran, Iran in 1998 and 2008, respectively. He joined Shahid Beheshti University, Tehran, Iran in 2008. His current research interests are focused on VLSI design automation and Automotive embedded system design. 